\title{
Mehrwert digitaler Checklisten
}

\author{
In Fertigung, Wartung und Montage setzen Unternehmen alles daran, produktiver zu werden. \\ Digitale Checklisten können dabei helfen - vorausgesetzt sie sind in eine digitale Plattform \\ eingebunden.
}

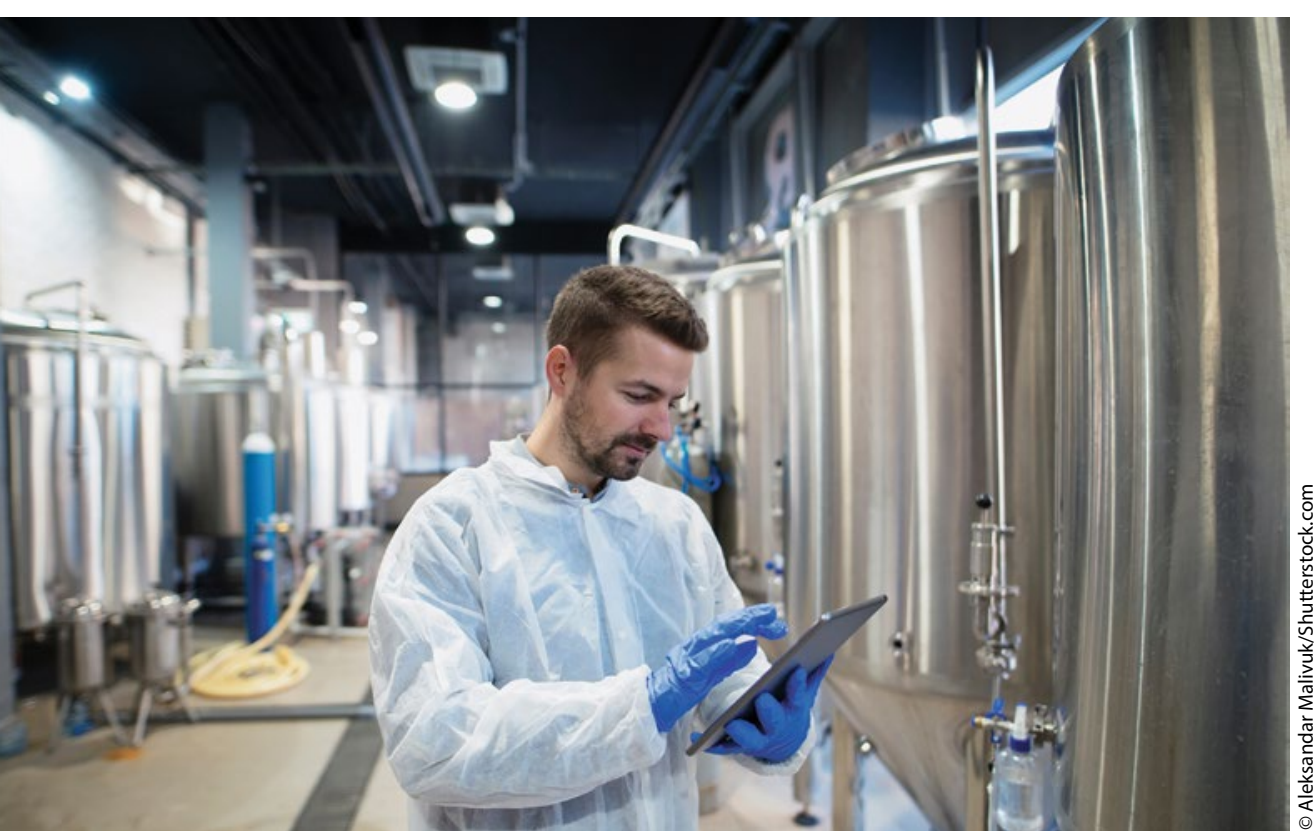

In der Industrie müssen Arbeiten so effizient wie nur möglich ausgeführt werden, möglichst niedrige Durchlaufzeiten sind das Ziel. Zu lange Rüstzeiten, aufwändiges Einarbeiten neuer Mitarbeiter oder unnötige Arbeitsschleifen stehen dem im Wege. Um solche Zeitfresser auszuradieren und Mitarbeitern möglichst klare Anweisungen an die Hand zu geben, arbeiten die meisten Fertigungsunternehmen mit Checklisten - viele noch in Papierform, immer mehr inzwischen digital. Doch die Evolution der Checkliste geht weiter: Wird sie nämlich in eine Plattform für vernetztes Arbeiten eingebunden, entsteht nochmals ein deutliches Plus an Effizienz. Der Anbieter Parsable erklärt, warum:

\section{Kein langes Suchen}

Unternehmen sehen digitale Checklisten oft nur als elektronische Variante des Papierbogens, als PDF mit Textfeldern und
Checkboxen. Dabei sind sie ihrem Zwilling auf Papier oder Whiteboard bereits klar überlegen. Sie lassen sich nicht nur auf mobilen Geräten einsetzen, sondern auch leichter und schneller durchsuchen - etwa nach dem nächsten Arbeitsauftrag. Noch besser, wenn der Mitarbeiter gar nicht erst suchen muss: Ist die App nämlich mit dem System zur Produktionsplanung gekoppelt, zeigt ihm ein Tablet oder ein anderes mobiles Gerät sofort seinen Arbeitsplan mit allen nötigen Schritten an. Das spart Zeit und hilft, dass alles reibungslos läuft.

\section{Vollständigkeit und Konsistenz sicherstellen}

Die Grundlage jeder Checkliste ist eine Standard Operating Procedure (SOP). Ist diese missverständlich oder unvollständig, wirft das Fragen auf. Dadurch entsteht nicht nur unnötiger Zeitverzug: Arbeiter erzielen dann möglicherweise auch nicht die nötige Qualität oder übersehen kri-
Digitale Plattformen für Connected Work tragen zu mehr Zeitersparnis, Qualität und einem reibungslosen Informationsfluss bei.

tische Sicherheitsprüfungen. Die Folgen können gravierend sein. Unternehmen müssen daher die SOPs kontinuierlich auf aktuellem Stand halten und verbessern. Hier bieten Systeme für „Connected Work“ grundsätzliche Vorteile, denn die Definition der Abläufe sowie die Konfiguration für die mobilen Geräte geschieht zentral. Doch lassen sie sich auch täglich aktualisieren und gezielt in den jeweiligen Unternehmensbereichen verteilen? Nur dann stellen Unternehmen sicher, dass ihre SOPs und Checklisten den lokalen Anforderungen jederzeit entsprechen. Wie wichtig schnelles Handeln sein kann, hat der Ausbruch von Covid-19 gezeigt.

\section{Aus Daten Erkenntnisse ziehen}

Viele Werkzeuge für digitale Checklisten sind interaktiv. Sie erlauben das Abhaken erledigter Tätigkeiten bis hin zum Eingeben von Messwerten. Eine gute Sache, aber leider nur die halbe Miete: Einer aktuellen 
Studie von Seagate zufolge bleiben nämlich $70 \%$ der in deutschen Unternehmen gesammelten Daten ungenutzt. Ein Grund dafür kann sein, dass diese nicht schnell genug für die weitere Analyse zur Verfügung stehen. Etwa, weil sie erst am Ende der Schicht und nur in statische PDF-Berichte ausgelesen werden. Zudem lassen sie sich oft nicht in Kontext mit anderen Daten - beispielsweise von Sensoren - setzen. Fehlen dann bei Problemen auch noch integrierte Warnmeldungen an Schichtleiter sowie Workflows mit den Sicherheits-Verantwortlichen, bleibt das Potenzial interaktiver Checklisten ungenutzt. Werden sie hingegen in Echtzeit ausgewertet und über eine zentrale Plattform für vernetztes Arbeiten weitergeleitet, fördern sie im Akutfall schnelles Reagieren. Auf lange Sicht tragen sie außerdem zu fundierten Prozessanalysen und nachhaltigen Verbesserungen bei.

\section{Ortsunabhängig zusammenarbeiten}

Ein Techniker, der auf ein Problem stößt, würde es seinen Kollegen oft gerne zeigen und sich mit ihnen beraten. Doch das geht längst nicht immer: etwa, wenn er sich gerade auf einem Rundgang durch eine weitläufige Industrieanlage befindet oder die Spezialisten für sein Thema über den ganzen Erdball verteilt sitzen. Eine Connected-Worker-Plattform wie die von Parsable überwindet diese örtlichen Gren- zen und macht den direkten Austausch leicht: Geteilte Fotos oder ein kurzes Video, ein paar Worte am Telefon oder per Chat helfen, das Problem direkt vor Ort gemeinsam zu beheben.

\section{Mitarbeiter aus- und fortbilden}

Digitale Checklisten leisten auch in der Ausbildung und Einarbeitung wertvolle Dienste. Sie helfen neuen Mitarbeitern sich zurechtzufinden und alle nötigen Arbeitsschritte in der richtigen Reihenfolge auszuführen. Plattformen für vernetztes Arbeiten stellen dafür obendrein ergänzende Videotutorials, Zeichnungen, Pläne und andere Anleitungen zur Verfügung. Gibt es Fragen, ermöglichen sie den direkten Austausch mit erfahrenen Kollegen oder einem Manager - unabhängig davon, wo sich diese gerade befinden. Das ist nicht nur beim Onboarding hilfreich, sondern lohnt sich immer dann, wenn Arbeitskräfte flexibler eingesetzt werden müssen.

\section{Zur kontinuierlichen Verbesserung beitragen}

In der Regel bieten Checklisten vor allem Orientierung, sehen aber kein Feedback zur Verbesserung des Arbeitsablaufs vor. Connected-Worker-Systeme halten diese Möglichkeit jedoch offen, denn sie erlau- ben Arbeitern detaillierte Rückmeldungen zu den erhaltenen Anleitungen. Wird eine Anregung zur Optimierung eines Arbeitsablaufs oder einer Tätigkeit übernommen, zeigen sich weitere Stärken des vernetzten Systems: Denn nun wird es zur Informationsdrehscheibe und Verteilerplattform. Entscheider geben neue Best Practices damit schnell an andere Schichten oder Unternehmensbereiche weiter - und haben zudem Gelegenheit, den oder die findigen Mitarbeitenden lobend hervorzuheben. Eine erhöhte Beteiligung an der Gestaltung von Abläufen und Prozessen fördert so die Selbstverantwortung und Identifikation mit dem Job.

Alles in allem tragen digitale Plattformen für Connected Work zu mehr Zeitersparnis, Qualität und einem reibungslosen Informationsfluss bei. Zusätzlich zu diesem Plus an Produktivität verbessern Unternehmen, die sich nicht mit einer einfachen Software für digitale Checklisten zufrieden geben, das Arbeitserlebnis sowie die Mitarbeiterzufriedenheit. //

\section{Kontakt}

\section{Parsable}

San Francisco (USA)

www.parsable.com

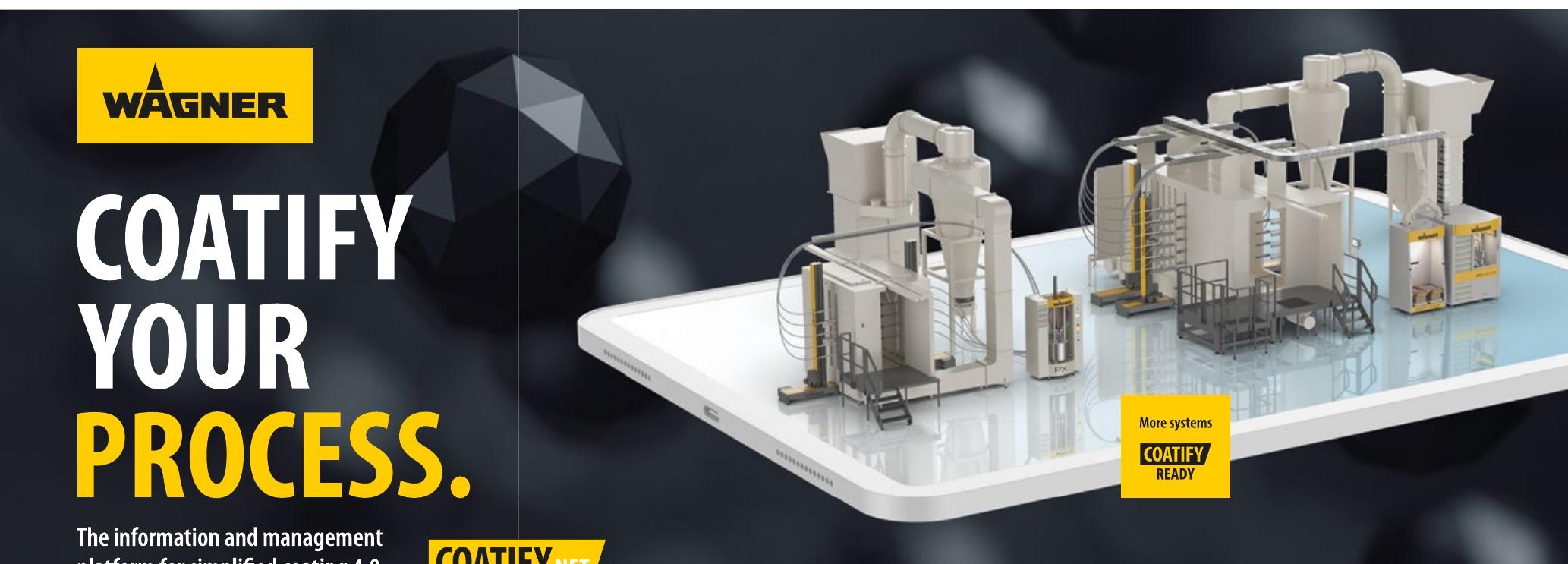

